\title{
May-Happen-in-Parallel Analysis for Asynchronous Programs with Inter-Procedural Synchronization
}

\author{
Elvira Albert, Samir Genaim, and Pablo Gordillo \\ Complutense University of Madrid (UCM), Spain
}

\begin{abstract}
A may-happen-in-parallel (MHP) analysis computes pairs of program points that may execute in parallel across different distributed components. This information has been proven to be essential to infer both safety properties (e.g., deadlock freedom) and liveness properties (termination and resource boundedness) of asynchronous programs. Existing MHP analyses take advantage of the synchronization points to learn that one task has finished and thus will not happen in parallel with other tasks that are still active. Our starting point is an existing MHP analysis developed for intra-procedural synchronization, i.e., it only allows synchronizing with tasks that have been spawned inside the current task. This paper leverages such MHP analysis to handle inter-procedural synchronization, i.e., a task spawned by one task can be awaited within a different task. This is challenging because task synchronization goes beyond the boundaries of methods, and thus the inference of MHP relations requires novel extensions to capture inter-procedural dependencies. The analysis has been implemented and it can be tried online.
\end{abstract}

\section{Introduction}

In order to improve program performance and responsiveness, many modern programming languages and libraries promote an asynchronous programming model, in which asynchronous tasks can execute concurrently with their caller tasks, until their callers explicitly wait for their completion. Our analysis is formalized for an abstract model that includes procedures, asynchronous calls, and future variables for synchronization $[8,7]$. In this model, a method call $\mathrm{m}$ on some parameters $\bar{x}$, written as $\mathrm{f}=\mathrm{m}(\bar{x})$, spawns an asynchronous task. Here, $\mathrm{f}$ is a future variable which allows synchronizing with the termination of the task executing $m$. The instruction await $f$ ? allows checking whether $m$ has finished, and blocks the execution of the current task if $m$ is still running. As concurrently-executing tasks interleave their accesses to shared memory, asynchronous programs are prone to concurrency-related errors [6]. Automatically proving safety and liveness properties still remains a challenging endeavor today.

MHP is an analysis of utmost importance to ensure both liveness and safety properties of concurrent programs. The analysis computes MHP pairs, which are pairs of program points whose execution might happen in parallel across different distributed components. In this fragment of code $f=m(..) ; \ldots$; await $f$ ?; the 
execution of the instructions of the asynchronous task m may happen in parallel with the instructions between the asynchronous call and the await. However, due to the await instruction, the MHP analysis is able to ensure that they will not run in parallel with the instructions after the await. This piece of information is fundamental to prove more complex properties: in [9], MHP pairs are used to discard unfeasible deadlock cycles; in [4], the use of MHP pairs allows proving termination and inferring the resource consumption of loops with concurrent interleavings. As a simple example, consider a procedure $\mathrm{g}$ that contains as unique instruction $y=-1$, where $y$ is a global variable. The following loop $\mathrm{y}=1$; while $(\mathrm{i}>0)\{\mathrm{i}=\mathrm{i}-\mathrm{y} ;\}$ might not terminate if $\mathrm{g}$ runs in parallel with it, since $\mathrm{g}$ can modify y to a negative value and the loop counter will keep on increasing. However, if we can guarantee that $\mathrm{g}$ will not run in parallel with this code, we can ensure termination and resource-boundedness for the loop.

This paper leverages an existing MHP analysis [3] developed for intra-procedural synchronization to the more general setting of inter-procedural synchronization. This is a fundamental extension because it allows synchronizing with the termination of a task outside the scope in which the task is spawned, as it is available in most concurrent languages. In the above example, if task $\mathrm{g}$ is awaited outside the boundary of the method that has spawned it, the analysis of [3] assumes that it may run in parallel with the loop and hence it fails to prove termination and resource boundedness. The enhancement to inter-procedural synchronization requires the following relevant extensions to the analysis:

1. Must-have-finished analysis (MHF): the development of a novel MHF analysis which infers inter-procedural dependencies among the tasks. Such dependencies allow us to determine that, when a task finishes, those that are awaited for on it must have finished as well. The analysis is based on using Boolean logic to represent abstract states and simulate corresponding operations. The key contribution is the use of logical implication to delay the incorporation of procedure summaries until synchronization points are reached. This is challenging in the analysis of asynchronous programs.

2. Local MHP phase: the integration of the above MHF information in the local phase of the original MHP analysis in which methods are analyzed locally, i.e., without taking transitive calls into account. This will require the use of richer analysis information in order to consider the inter-procedural dependencies inferred in point 1 above.

3. Global MHP phase: the refinement of the global phase of the MHP analysis -where the information of the local MHP analysis in point 2 is composed-in order to eliminate spurious MHP pairs which appear when inter-procedural dependencies are not tracked. This will require to refine the way in which MHP pairs are computed.

We have implemented our approach in SACO [2], a static analyzer for concurrent objects which is able to infer the aforementioned liveness and safety properties. The system can be used online at http://costa.ls.fi.upm.es/saco/web, where the examples used in the paper are also available. 


\section{Language}

Our analysis is formalized for an abstract model that includes procedures, asynchronous calls, and future variables $[8,7]$. It also includes conditional and loop constructs, however, conditions in these constructs are simply non-deterministic choices. Developing the analysis at such abstract level is convenient [11], since the actual computations are simply ignored in the analysis and what is actually tracked is the control flow that originates from asynchronously calling methods and synchronizing with their termination. Our implementation, however, is done for the full concurrent object-oriented language ABS [10] (see Sec. 6).

A program $P$ is a set of methods that adhere to the following grammar:

$$
\begin{aligned}
& M::=m(\bar{x})\{s\} \quad s::=\epsilon \mid b ; s \\
& b \quad::=\text { if }(*) \text { then } s_{1} \text { else } s_{2} \mid \text { while }(*) \text { do } s|y=m(\bar{x})| \text { await } x ? \mid \text { skip }
\end{aligned}
$$

Here all variables are future variables, which are used to synchronize with the termination of the called methods. Those future variables that are used in a method but are not in its parameters are the local future variables of the method (thus we do not need any special instruction for declaring them). In loops and conditions, the symbol $*$ stands for non-deterministic choice (true or false). The instruction $y=m(\bar{x})$ creates a new task which executes method $m$, and binds the future variable $y$ with this new task so we can synchronize with its termination later. Inter-procedural synchronization is realized in the language by passing future variables as parameters, since the method that receives the future variable can await for the termination of the associated task (created outside its scope). For simplifying the presentation, we assume that method parameters are not modified inside each method. For a method $m$, we let $P_{m}$ be the set of its parameters, $L_{m}$ the set of its local variables, and $V_{m}=P_{m} \cup L_{m}$.

The instruction await $x$ ? blocks the execution of the current task until the task associated with $x$ terminates. Instruction skip has no effect, it is simply used when abstracting from a richer language, e.g., ABS in our case, to abstract instructions such as assignments. Programs should include a method main from which the execution (and the analysis) starts. We assume that instructions are labeled with unique identifiers that we call program points. For if and while the identifier refers to the corresponding condition. We also assume that each method has an exit program point $\ell_{m}$. We let ppoints $(m)$ and ppoints $(P)$ be the sets of program points of method $m$ and program $P$, resp., $I_{\ell}$ be the instruction at program point $\ell$, and pre $(\ell)$ be the set of program points preceding $\ell$.

Next we define a formal (interleaving) operational semantics for our language. A task is of the form $t s k(t i d, l, s)$ where tid is a unique identifier, $l$ is a mapping from local variables and parameters to task identifiers, and $s$ is a sequence of instructions. Local futures are initialized to $\perp$. A state $S$ is a set of tasks that are executing in parallel. From a state $S$ we can reach a state $S^{\prime}$ in one execution step, denoted $S \leadsto S^{\prime}$, if $S$ can be rewritten using one of the derivation rules of Fig. 1 as follows: if the conclusion of the rule is $A \leadsto B$ such that $A \subseteq S$ and the premise holds, then $S^{\prime}=(S \backslash A) \cup B$. The meaning of the derivation rules is quite straightforward: (SKIP) advances the execution of the corresponding task to the next instruction; (IF) nondeterministically chooses between one of 
(IF) $\frac{b \equiv \text { if }(*) \text { then } s_{1} \text { else } s_{2} \text {, set } s^{\prime} \text { non-deterministically to } s_{1} ; s \text { or } s_{2} ; s}{t s k(t i d, l, b ; s) \sim t s k\left(t i d, l, s^{\prime}\right)}$

(LOOP) $\frac{b \text { while }(*) \text { do } s_{1} \text {, set } s^{\prime} \text { non-deterministically to } s_{1} ; b ; s \text { or } s}{t s k(t i d, l, b ; s) \sim t s k\left(t i d, l, s^{\prime}\right)}$

$(\mathrm{CALL}) \frac{\bar{z} \text { are the formal parameters of } m, t i d^{\prime} \text { is a fresh id, } l^{\prime}=\left\{z_{i} \mapsto l\left(x_{i}\right)\right\}}{t s k(t i d, l, y=m(\bar{x}) ; s) \sim \operatorname{tsk}\left(t i d, l\left[y \mapsto t i d^{\prime}\right], s\right), t s k\left(\operatorname{tid}^{\prime}, l^{\prime}, \operatorname{body}(m)\right)}$

$($ AWAIT $) \quad \frac{l(x)=t i d^{\prime}}{t s k(t i d, l, \text { await } x ? ; s), t s k\left(t i d^{\prime}, l^{\prime}, \epsilon\right) \sim t s k(t i d, l, s), t s k\left(t i d^{\prime}, l^{\prime}, \epsilon\right)}$

Fig. 1. Derivation Rules

the branches; (LOOP) nondeterministically chooses between executing the loop body or advancing to the instruction after the loop; (CALL) creates a new task with a fresh identifier $t i d^{\prime}$, initializes the formal parameters $\bar{z}$ of $m$ to those of the actual parameters $\bar{x}$, sets future variable $y$ in the calling task to $t i d^{\prime}$, so one can synchronize with its termination later (other local futrures are assumed to be $\perp$ ); and (AWAIT) advances to the next instruction if the task associated to $x$ has terminated already. Note that when a task terminates, it does not disappear from the state but rather its sequence of instructions remains empty.

An execution is a sequence of states $S_{0} \leadsto S_{1} \leadsto \cdots \leadsto S_{n}$, sometimes denoted as $S_{0} \sim^{*} S_{n}$, where $S_{0}=\{t s k(0, l, b o d y($ main $))\}$ is an initial state which includes a single task that corresponds to method main, and $l$ is an empty mapping. At each step there might be several ways to move to the next state depending on the task selected, and thus executions are nondeterministic.

In what follows, given a task $t s k(t i d, l, s)$, we let $p p(s)$ be the program point of the first instruction in $s$. When $s$ is an empty sequence, $p p(s)$ refers to the exit program point of the corresponding method. Given a state $S$, we define its set of MHP pairs, i.e., the set of program points that execute in parallel in $S$, as $\mathcal{E}(S)=\left\{\left(p p\left(s_{1}\right), p p\left(s_{2}\right)\right) \mid t s k\left(t i d_{1}, l_{1}, s_{1}\right), t s k\left(t i d_{2}, l_{2}, s_{2}\right) \in S, t i d_{1} \neq t i d_{2}\right\}$. The set of MHP pairs for a program $P$ is then defined as the the set of MHP pairs of all reachable states, namely $\mathcal{E}_{P}=\cup\left\{\mathcal{E}\left(S_{n}\right) \mid S_{0} \sim^{*} S_{n}\right\}$.

Example 1. Fig. 2 shows some simple examples in our language. Methods $\mathrm{m}_{1}, \mathrm{~m}_{2}$ and $\mathrm{m}_{3}$ are main methods and the remaining ones are auxiliary. Let us consider some steps of one possible derivation from $\mathrm{m}_{2}$ :

$$
\begin{aligned}
& \mathbf{S}_{\mathbf{0}} \equiv \operatorname{tsk}\left(0, \emptyset, \operatorname{body}\left(\mathrm{m}_{2}\right)\right) \sim^{*} \mathbf{S}_{1} \equiv \operatorname{tsk}(0,[x \mapsto 1],\{16, \ldots\}), \operatorname{tsk}(1, \emptyset, \operatorname{body}(\mathrm{f})) \sim^{*} \\
& \mathbf{S}_{\mathbf{2}} \equiv \operatorname{tsk}(0,[x \mapsto 1, z \mapsto 2],\{18, \ldots\}), \operatorname{tsk}(1, \emptyset, \operatorname{body}(\mathrm{f})), \operatorname{tsk}(2,[w \mapsto 1], \operatorname{body}(\mathrm{g})) \sim^{*} \\
& \mathbf{S}_{\mathbf{3}} \equiv \operatorname{tsk}(0,[x \mapsto 1, z \mapsto 2],\{19, \ldots\}), \operatorname{tsk}(1, \emptyset, \epsilon), \operatorname{tsk}(2,[w \mapsto 1], \operatorname{body}(\mathrm{g})) \sim^{*} \\
& \mathbf{S}_{\mathbf{4}} \equiv \operatorname{tsk}(0,[x \mapsto 1, z \mapsto 2],\{20, \ldots\}), \operatorname{tsk}(1, \emptyset, \epsilon), \operatorname{tsk}(2,[w \mapsto 1], \epsilon) \sim \ldots
\end{aligned}
$$

In $S_{1}$ we execute until the asynchronous call to $f$ which creates a new task identified as 1 and binds $\times$ to this new task. In $S_{2}$ we have executed the skip and the asynchronous invocation to $\mathrm{g}$ that adds in the new task the binding of the formal parameter $w$ to the task identified as 1 . In $S_{3}$ we proceed with the execution of the instructions in $\mathrm{m}_{2}$ until reaching the await that blocks this task until g terminates. Also, in $S_{3}$ we have executed entirely $\mathrm{f}$ (denoted by $\epsilon$ ). $S_{4}$ 


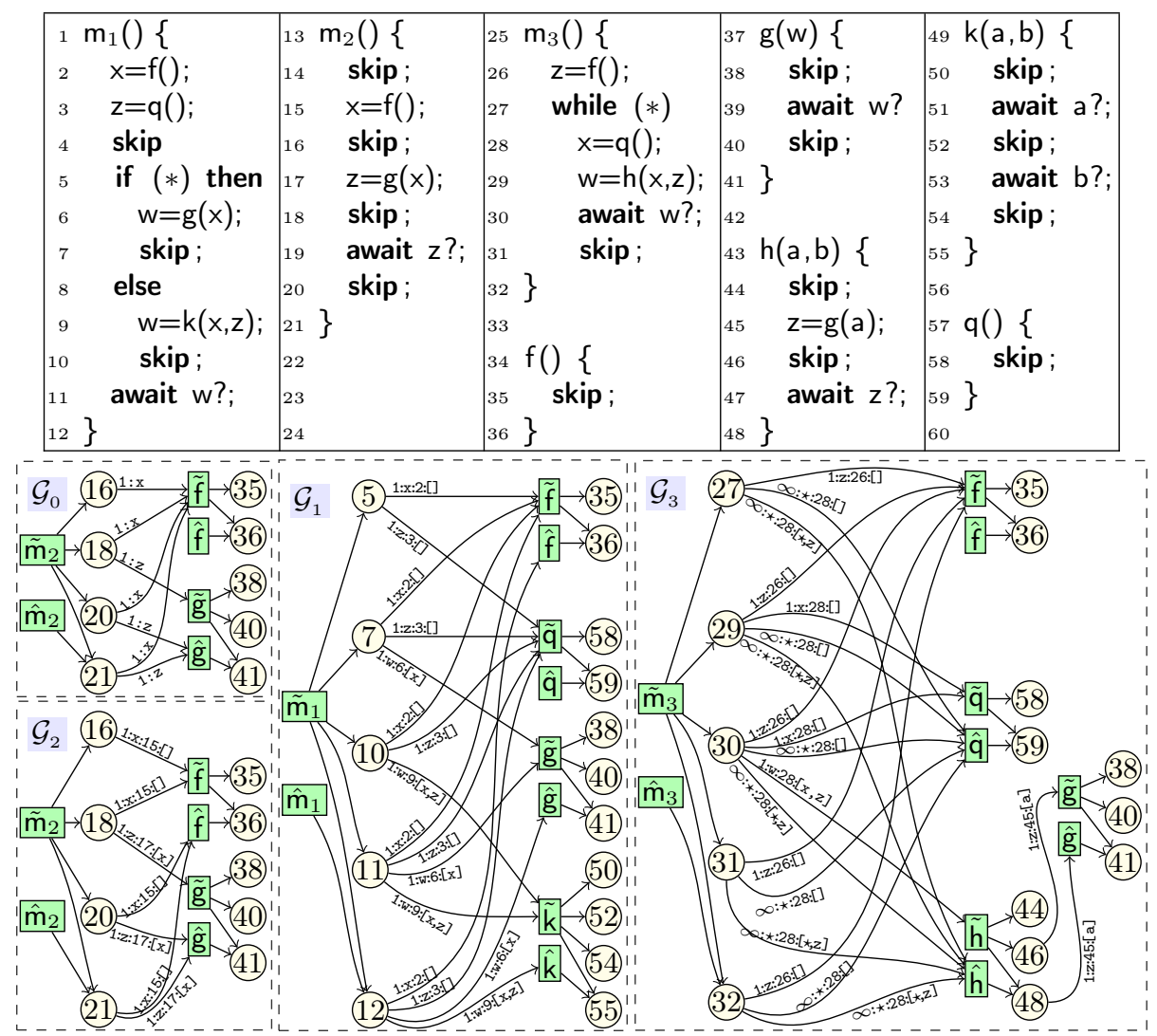

Fig. 2. (TOP) Examples for MHP analysis $\left(\mathrm{m}_{1}, \mathrm{~m}_{2}, \mathrm{~m}_{3}\right.$ are main methods). (BOTTOM) MHP graph $\mathcal{G}_{i}$ corresponds to analyzing $\mathrm{m}_{i}$, and $\mathcal{G}_{0}$ to analyzing $\mathrm{m}_{2}$ as in [3].

proceeds with the execution of $g$ whose await can be executed since task 1 is at its exit point $\epsilon$. We have the following MHP pairs in this fragment of the derivation, among many others: from $S_{1}$ we have $(16,35)$ that captures that the first instruction of $\mathrm{f}$ executes in parallel with the instruction 16 of $\mathrm{m} 2$, from $S_{2}$ we have $(18,35)$ and $(18,38)$. The important point is that we have no pair $(20,35)$ since when the await at L19 executes at $S_{4}$, it is guaranteed that $\mathrm{f}$ has finished. This is due to the inter-procedural dependecy at L39 of $g$ where the task $f$ is awaited: variable $x$ is passed as argument to $g$, which allows $g$ to synchronize with the termination of $f$ at L39 even if $f$ was called in a different method.

\section{An Informal Account of our Method}

In this section, we provide an overview of our method by explaining the analysis of $\mathrm{m}_{2}$. Our goal is to infer precise MHP information that describes, among others, the following representative cases: (1) any program point of $\mathrm{g}$ cannot run in parallel with L20, because at L19 method $\mathrm{m}_{2}$ awaits for $\mathrm{g}$ to terminate; (2) L35 cannot run in parallel with L20, since when waiting for the termination of $g$ at L19 we know that $\mathrm{f}$ must-have-finished as well due to the dependency relation 
that arises when $m_{2}$ implicitly waits for the termination of $f$; and (3) L35 cannot run in parallel with L40, because $\mathrm{f}$ must-have-finished due to the synchronization on the local future variable $w$ at L39 that refers to future variable $x$ of $m_{2}$.

Let us first informally explain which MHP information the analysis of [3] is able to infer for $\mathrm{m}_{2}$, and identify the reasons because it fails to infer some of the desired information. The analysis of [3] is carried out in two phases: (1) each method is analyzed separately to infer local MHP information; and (2) the local information is used to construct a global MHP graph from which MHP pairs are extracted by checking reachability conditions among the nodes.

The local analysis infers, for each program point, a multiset of MHP atoms where each atom describes a task that might be executing in parallel when reaching that program point, but only considering tasks that have been invoked locally within the analyzed method. An atom of the form $x: \tilde{m}$ indicates that there might be an active instance of $m$ executing at any of its program points, and is bounded to the future variable $x$. An atom of the form $x: \hat{m}$ differs from the previous one in that $m$ must be at its exit program point, i.e., has finished executing already. For method $\mathrm{m}_{2}$, the local MHP analysis infers, among others, $\{x: \tilde{f}\}$ for L16, $\{x: \tilde{f}, z: \tilde{g}\}$ for L18, and $\{x: \tilde{f}, z: \hat{g}\}$ for L20 and L21, because $g$ has been awaited locally. Observe that the sets of L20 and L21 include $x: \tilde{f}$ and not $\mathrm{x}: \hat{\mathrm{f}}$, although we know that method $\mathrm{f}$ has finished already when reaching L20 and L21 (since $\mathrm{g}$ has finished). This information cannot be inferred by the local analysis of [3] since it is applied to each method separately, ignoring (a) transitive (non-local) calls and $(b)$ inter-procedural synchronizations. In the sequel we let $\Psi_{\ell}$ be the result of the local MHP analysis for program point $\ell$.

In the second phase, the analysis of [3] builds the MHP graph whose purpose is to capture MHP relations due to transitive calls (point (a) above). The graph $\mathcal{G}_{0}$ depicted in Fig. 2 for $\mathrm{m}_{2}$ is constructed as follows: (1) every program point $\ell$ contributes a node labeled with $\ell$ - for simplicity we include only program points of interest; (2) every method $m$ contributes two nodes $\tilde{m}$ and $\hat{m}$, where $\tilde{m}$ is connected to all program point nodes of $m$, to indicate that an active method can be executing at any of its program points, and $\hat{m}$ is connected only to the exit program point of $m$; and (3) if $x: \tilde{m}$ (resp. $x: \hat{m}$ ) is an atom of $\Psi_{\ell}$ with multiplicity $i$, we create an edge from $\ell$ to $\tilde{m}$ (resp. $\hat{m}$ ) and label it with $i: x$. Note that $i$ is the multiplicity of the edge, i.e., we could copy the edge $i$ times instead.

Roughly, the MHP pairs are obtained from $\mathcal{G}_{0}$ using the following principle: program points $\left(\ell_{1}, \ell_{2}\right)$ might execute in parallel if there is a path from $\ell_{1}$ to $\ell_{2}$ or vice versa (direct MHP pair); or if there is a program point $\ell_{3}$ such that there are paths from $\ell_{3}$ to $\ell_{1}$ and to $\ell_{2}$ (indirect MHP pair), and the first edge of both paths is labeled with two different future variables. When two paths are labeled with the same future variable, it is because there is a disjunction (e.g., from an if-then-else) and only one of the paths might actually occur. Applying this principle to $\mathcal{G}_{0}$, we can conclude that L20 cannot execute in parallel with any program point of $\mathrm{g}$, which is precise as expected, and that L20 can execute in parallel with L35 which is imprecise. This imprecision is attributed to the fact that the MHP analysis of [3] does not track inter-method synchronizations. 
To overcome the imprecision, we develop a must-have-finished analysis that captures inter-method synchronizations, and use it to improve the two phases of [3]. This analysis would infer, for example, that "when reaching L40, it is guaranteed that whatever task bounded to w has finished already", and that "when reaching L20, it is guaranteed that whatever tasks bounded to $x$ and $z$ have finished already". By having this information at hand, the first phase of [3] can be improved as follows: when analyzing the effect of await $z$ ? at L20, we change the status of both $g$ and $f$ to finished, because we know that any task bounded $z$ and $x$ has finished already. This will require to enrich the information of the MHP atoms as follows: an MHP atom will be of the form $y: \ell: \tilde{m}(\bar{x})$ or $y: \ell: \hat{m}(\bar{x})$, where the new information $\ell$ and $\bar{x}$ are the calling site and the parameters passed to $m$. In summary, the modified first phase will infer $\{x: 15: \tilde{f}()\}$ for L16, $\{\mathrm{x}: 15: \tilde{\mathrm{f}}(), \mathrm{z}: 17: \tilde{\mathrm{g}}(\mathrm{x})\}$ for L18, and $\{\mathrm{x}: 15: \hat{\mathrm{f}}(), \mathrm{z}: 17: \hat{\mathrm{g}}(\mathrm{x})\}$ for L20 and L21.

In the second phase of the analysis: (i) the construction of the MHP graph is modified to use the new local MHP information; and (ii) the principle used to extract MHP pairs is modified to make use to the must-have-finished information. The new MHP graph constructed for $\mathrm{m}_{2}$ is depicted in Fig. 2 as $\mathcal{G}_{2}$. Observe that the labels on the edges include the new information available in the MHP atoms. Importantly, the spurious MHP information that is inferred by [3] is not included in this graph: (1) in contrast to $\mathcal{G}_{0}, \mathcal{G}_{2}$ does not include edges from nodes 20 and 21 to $\tilde{f}$, but to $\hat{f}$. This implies that L35 cannot run in parallel with L20 or L21; (2) in $\mathcal{G}_{2}$, we still have paths from 18 to 35 and 40, which means, if the old principle for extracting MHP pairs is used, that L35 and L40 might happen in parallel. The main point is that, using the labels on the edges, we know that the first path uses a call to $f$ that is bounded to $x$, and that this same $x$ is passed to $g$, using the parameter $w$, in the first edge of the second path. Now since the must-have-finished analysis tell us that at L40 any task bounded $w$ is finished already, we conclude that $f$ must be at its exit program point and thus this MHP pair is spurious (because L35 is not an exit program point).

\section{Must-Have-Finished Analysis}

In this section we present a novel inter-procedural Must-Have-Finished (MHF) analysis that can be used to compute, for each program point $\ell$, a set of finished future variables, i.e., whenever $\ell$ is reached those variables are either not bounded to any task (i.e., have value $\perp$ ) or their bounded tasks are guaranteed to have terminated. We refer to such sets as MHF sets.

Example 2. The following are MHF sets for the program points of Fig. 2:

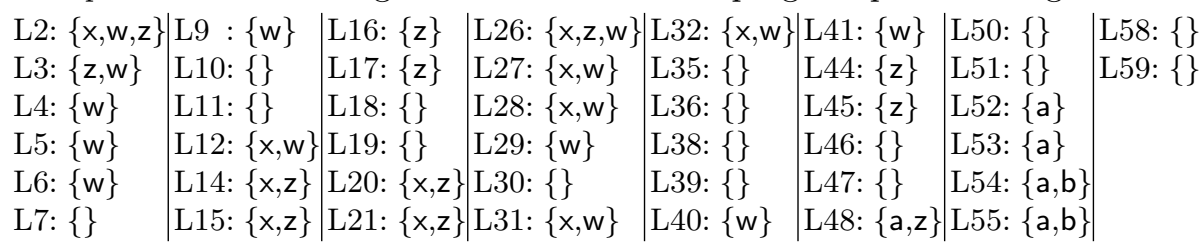

At program points that correspond to method entries, all local variables (but not the parameters) are finished since they point to no task. For g: at L38 and 
L39 no task is guaranteed to have finished, because the task bounded to w might be still executing; at L40 and L41, since we passed through await w? already, it is guaranteed that $w$ is finished. For k: at L50 and L51 no task is guaranteed to have finished; at L52 and L53 a is finished since we already passed through await a?; and at L54 and L55 both a and b are finished. For $\mathrm{m}_{1}$ : at L12 both w and $x$ are finished. Note that $w$ is finished because of await $w$ ?, and $x$ is finished due to the implicit dependency between the termination of $x$ and $w$.

\subsection{Definition of MHF}

By carefully examining the MHF sets of Ex. 2, we can see that an analysis that simply tracks MHF sets would be imprecise. For example, since the MHF set at L11 is empty, the only information we can deduce for L12 is that $w$ is finished. To deduce that $\mathrm{x}$ is finished we must track the implicit dependency between $\mathrm{w}$ and $\mathrm{x}$. Next we define a more general MHF property that captures such dependencies, and from which we can easily compute the MHF sets.

Definition 1. Given a program point $\ell \in \operatorname{ppoints}(P)$, we let $\mathcal{F}(\ell)=\left\{f\left(S_{i}, l\right) \mid\right.$ $S_{0} \sim^{*} S_{i}, \operatorname{tsk}($ tid $\left., l, s) \in S_{i}, p p(s)=\ell\right\}$ where $f(S, l)=\{x \mid x \in \operatorname{dom}(l), l(x)=$ $\left.\perp \vee\left(l(x)=t i d^{\prime} \wedge t s k\left(t i d^{\prime}, l^{\prime}, \epsilon\right) \in S\right)\right\}$.

Intuitively, $f(S, l)$ is the set of all future variables, from those defined in $l$, whose corresponding tasks are finished in $S$. The set $\mathcal{F}(\ell)$ considers all possible ways of reaching $\ell$, and for each one it computes a corresponding set $f(S, l)$ of finished future variables. Thus, $\mathcal{F}(\ell)$ describes all possible sets of finished future variables when reaching $\ell$. The set of all finished future variables at $\ell$ is then defined as $\operatorname{mhf}(\ell)=\cap\{F \mid F \in \mathcal{F}(\ell)\}$, i.e., the intersection of all sets in $\mathcal{F}(\ell)$.

Example 3. The values of $\mathcal{F}(\ell)$ for selected program points from Fig. 2 are:

\begin{tabular}{|c|c|c|}
\hline & & \\
\hline L5 : $\{\{w, x, z\},\{w, z\},\{w$, & L31: $\{\{w, x, z\},\{w, x\}\}$ & $:\{\{a, z\},\{a$ \\
\hline L11: $\{\{w, x, z\},\{w, x\},\{x, z$ & L32: $\{\{w, x, z\},\{w, x\}\}$ & $\{a, b, z\},\{a, b\},\{b\}$ \\
\hline L12: $\{\{\mathrm{x}, \mathrm{w}, \mathrm{z}\},\{\mathrm{w}, \mathrm{x}\}\}$ & L35: $\{\{\}\}$ & L48: $\{\{a, z\},\{a, b, z\}\}$ \\
\hline L20: $\{\{x, z\}\}$ & L38: $\{\{w\},\{\}\}$ & $\mathrm{L} 52:\{\{\mathrm{a}\},\{\mathrm{a}, \mathrm{b}\}\}$ \\
\hline $\mathrm{L} 27:\{\{w, x, z\},\{w, x\}\}$ & L40: $\{\{w\}\}$ & $\mathrm{L} 54:\{\{a, b\}\}$ \\
\hline L30: $\{\{w, x, z\},\{w, x\},\{x, z\},\{x\},\{z\},\{\}\}$ & & L58: $\{\{\}\}$ \\
\hline
\end{tabular}

In L5 different sets arise by considering all possible orderings in the execution of tasks $\mathrm{f}$, $\mathrm{q}$ and $\mathrm{m}_{1}$, but $\operatorname{mhf}(L 5)=\{\mathrm{w}\}$. Note that for any $F \in \mathcal{F}(11)$, if $\mathrm{w} \in F$ then $\mathrm{x} \in F$, which means that if $\mathrm{w}$ is finished at L11, then $\mathrm{x}$ must finish too.

\subsection{An Analysis to Infer MHF Sets}

Our goal is to infer $\operatorname{mhf}(\ell)$, or a subset of it, for each $\ell \in \operatorname{ppoints}(P)$. Note that any set $X$ that over-approximates $\mathcal{F}(\ell)$, i.e., $\mathcal{F}(\ell) \subseteq X$, can be used to compute a subset of $\operatorname{mhf}(\ell)$, because $\cap\{F \mid F \in X\} \subseteq \cap\{F \mid F \in \mathcal{F}(\ell)\}$. In the rest of this section we develop an analysis to over-approximate $\mathcal{F}(\ell)$. We will use Boolean formulas, whose models naturally represent MHF sets, and, moreover, Boolean connectives smoothly model the abstract execution of the different instructions. 
An MHF state for the program points of a method $m$ is a propositional formula $\Phi: V_{m} \mapsto\{$ true, false $\}$ of the form $\vee_{i} \wedge_{j} c_{i j}$, where an atomic proposition $c_{i j}$ is either $x$ or $y \rightarrow x$ such that $x \in V_{m} \cup\{$ true, false $\}$ and $y \in L_{m}$. Intuitively, an atomic proposition $x$ states that $x$ is finished, and $y \rightarrow x$ states that if $y$ is finished then $x$ is finished as well. Note that we do not allow the parameters of $m$ to appear in the premise of an implication (we require $y \in L_{m}$ ). When $\Phi$ does not include any atomic proposition of the form $y \rightarrow x$ we call it monotone. Recall that $\sigma \subseteq V_{m}$ is a model of $\Phi$, iff an assignment that maps variables from $\sigma$ to true and other variables to false is a satisfying assignment for $\Phi$. The set of all models of $\Phi$ is denoted $\llbracket \Phi \rrbracket$. The set of all MHF states for $m$, together with the formulas true and false, is denoted $\mathcal{A}_{m}$.

Example 4. Assume $V_{m}=\{x, y, z\}$. The Boolean formula $x \vee y$ states that either $x$ or $y$ or both are finished, and that $z$ can be in any status. This information is precisely captured by the models $\llbracket x \vee y \rrbracket=\{\{x\},\{y\},\{x, y\},\{x, z\},\{y, z\},\{x, y, z\}\}$. The Boolean formula $z \wedge(x \rightarrow y)$ states that $z$ is finished, and if $x$ is finished then $y$ is finished. This is reflected in $\llbracket z \wedge(x \rightarrow y) \rrbracket=\{\{z\},\{z, y\},\{z, x, y\}\}$ since $z$ belongs to all models, and any model that includes $x$ includes $y$ as well. The formula false means that the corresponding program point is not reachable. The following MHF states correspond to some selected program points from Fig. 2:

$\Phi_{5}: \mathrm{w} \quad\left|\Phi_{12}: \mathrm{w} \wedge \mathrm{x}\right| \Phi_{27}: \mathrm{w} \wedge \mathrm{x}\left|\Phi_{31}: \mathrm{w} \wedge \mathrm{x}\right| \Phi_{35}:$ true $\left|\Phi_{40}: \mathrm{w} \quad\right| \Phi_{48}: \mathrm{a} \wedge \mathrm{z} \mid \Phi_{54}: \mathrm{a} \wedge \mathrm{b}$

$\Phi_{11}: \mathrm{w} \rightarrow \mathrm{x}\left|\Phi_{20}: \mathrm{x} \wedge \mathrm{z}\right| \Phi_{30}: \mathrm{w} \rightarrow \mathrm{x}\left|\Phi_{32}: \mathrm{w} \wedge \mathrm{x}\right| \Phi_{38}: \operatorname{true}\left|\Phi_{46}: \mathrm{z} \rightarrow \mathrm{a}\right| \Phi_{52}: \mathrm{a} \quad \mid \Phi_{58}:$ true

Note that the models $\llbracket \Phi_{\ell} \rrbracket$ coincide with $\mathcal{F}(\ell)$ from Ex. 3.

Now that is clear how Boolean formulas represent the desired MHF information, we proceed to explain how the execution of the different instructions can be modeled with Boolean formulas. Let us first define some auxiliary operations. Given a variable $x$ and an MHF state $\Phi \in \mathcal{A}_{m}$, we let $\exists x . \Phi=\Phi[x \mapsto$ true $] \vee \Phi[x \mapsto$ false $]$, i.e., this operation eliminates variable $x$ from (the domain of) $\Phi$. Note that $\exists x . \Phi \in \mathcal{A}_{m}$ and that $\llbracket \Phi \rrbracket \models \llbracket \exists x . \Phi \rrbracket$. For a tuple of variables $\bar{x}$ we let $\exists \bar{x} . \Phi$ be $\exists x_{1} . \exists x_{2} \ldots . \exists x_{n} . \Phi$, i.e., eliminate all variables $\bar{x}$ from $\Phi$. We also let $\bar{\exists} \bar{x} . \Phi$ stand for eliminating all variables but $\bar{x}$ from $\Phi$. Note that if $\Phi \in \mathcal{A}_{m}$ and monotone, and $x \in L_{m}$, then $x \rightarrow \Phi$ is a formula in $\mathcal{A}_{m}$ as well.

Given a program point $\ell$, an MHF state $\Phi_{\ell}$, and an instruction to execute $I_{\ell}$, our aim is to compute a new MHF state, denoted $\mu\left(I_{\ell}\right)$, that represents the effect of executing $I_{\ell}$ within $\Phi_{\ell}$. If $I_{\ell}$ is skip, then clearly $\mu\left(I_{\ell}\right) \equiv \Phi_{\ell}$. If $I_{\ell}$ is an await $x$ ? instruction, then $\mu\left(I_{\ell}\right)$ is $x \wedge \Phi_{\ell}$, which restricts the MHF state of $\Phi_{\ell}$ to those cases (i.e., models) in which $x$ is finished. If $I_{\ell}$ is a call $y=m(\bar{x})$, where $m$ is a method with parameters named $\bar{z}$, and, at the exit program point of $m$ we know that the MHF state $\Phi_{\ell_{m}}$ holds, then $\mu\left(I_{\ell}\right)$ is computed as follows:

- We compute an MHF state $\Phi_{m}$ that describes "what happens to tasks bounded to $\bar{x}$ when $m$ terminates". This is done by restricting $\Phi_{\ell_{m}}$ to the method parameters, and then renaming the formal parameters $\bar{z}$ to the actual parameters $\bar{x}$, i.e., $\Phi_{m}=\left(\bar{\exists} \bar{z} \cdot \Phi_{\ell_{m}}\right)[\bar{z} / \bar{x}]$, where $[\bar{z} / \bar{x}]$ denotes the renaming.

- Now assume that $\xi$ is a new (future) variable to which $m$ is bounded. Then $\xi \rightarrow \Phi_{m}$ states that "when $m$ terminates, $\Phi_{m}$ must hold". Note that it says nothing about $\bar{x}$ if $m$ has not terminated yet. 
- Next we add $\xi \rightarrow \Phi_{m}$ to $\Phi_{\ell}$, eliminate (old) $y$ since the variable is rewritten, and rename $\xi$ to (new) $y$. Note that we use $\xi$ as a temporary variable just not to conflict with the old value of $y$.

The above reasoning is equivalent to $\left(\exists y \cdot\left(\Phi_{\ell} \wedge\left(\xi \rightarrow\left(\bar{\exists} \bar{z} \cdot \Phi_{\ell_{m}}\right)[\bar{z} / \bar{x}]\right)\right)[\xi / y]\right.$, and is denoted by $\oplus\left(\Phi_{\ell}, y, \Phi_{\ell_{m}}, \bar{x}, \bar{z}\right)$.

Example 5. Let $\Phi_{11}=\mathrm{x} \rightarrow \mathrm{w}$ be the MHF state at L11. The effect of executing $I_{11}$, i.e., await w?, within $\Phi_{11}$ should eliminate all models that do not include $\mathrm{w}$. This is done using $\mathrm{w} \wedge \Phi_{11}$ which results in $\Phi_{12}=\mathrm{w} \wedge \mathrm{x}$. Now let $\Phi_{29}=\mathrm{w}$ be the MHF state at L29. The effect of executing the instruction at L29, i.e., $\mathrm{w}=\mathrm{h}(\mathrm{x}, \mathrm{z})$, within $\Phi_{29}$ is defined as $\oplus\left(\Phi_{29}, \mathrm{w}, \Phi_{48},\langle\mathrm{x}, \mathrm{z}\rangle,\langle\mathrm{a}, \mathrm{b}\rangle\right)$ and computed as follows: (1) we restrict $\Phi_{48}=\mathrm{a} \wedge \mathrm{z}$ to the method parameters $\langle\mathrm{a}, \mathrm{b}\rangle$, which results in a; (2) we rename the formal parameters $\langle a, b\rangle$ to the actual ones $\langle x, z\rangle$ which results in $\Phi_{\mathrm{h}}=\mathrm{x} ;(3)$ we compute $\exists \mathrm{w} .\left(\Phi_{29} \wedge\left(\xi \rightarrow \Phi_{\mathrm{h}}\right)\right)$, which results in $\xi \rightarrow \mathrm{x}$; and finally (4) we rename $\xi$ to w which results in $\Phi_{30}=\mathrm{w} \rightarrow \mathrm{x}$.

Next we define our MHF analysis by means of data-flow equations, whose solutions associate to each $\ell \in$ ppoints $(P)$ an MHF state $\Phi_{\ell}$ that over-approximates $\mathcal{F}(\ell)$, i.e., $\mathcal{F}(\ell) \subseteq \llbracket \Phi_{\ell} \rrbracket$.

Definition 2. The set of $\mathcal{H}_{P}$ of equations for a program $P$ is defined as follows:

1. For each $\ell \in \operatorname{ppoints}(P)$ such that $\ell$ is not an entry program point, we generate the equation $\Phi_{\ell}=\vee\left\{\mu\left(\ell^{\prime}\right) \mid \ell^{\prime} \in \operatorname{pre}(\ell)\right\}$;

2. For each $\ell \in \operatorname{ppoints}(P)$ such that $\ell$ is an entry program point for method $m \neq$ main, let $\left\{\ell_{1}, \ldots, l_{k}\right\}$ be the program points in which $m$ is called, we generate $\Phi_{\ell}=\left(\vee\left\{T F\left(\Phi_{\ell_{i}}\right) \mid \ell_{i} \in\left\{\ell_{1}, \ldots, \ell_{k}\right\}\right\}\right) \wedge\left(\wedge\left\{x \mid x \in L_{m}\right\}\right)$

3. If $\ell$ is the entry program point of main, we generate $\Phi_{\ell}=\wedge\left\{x \mid x \in L_{\text {main }}\right\}$.

Let us explain the meaning of each equation in the above defintion: (1) when $\ell$ is not a method entry, we consider each program point $\ell^{\prime}$ that immediately precedes $\ell$, compute the effect $\mu\left(\ell^{\prime}\right)$ of executing $I_{\ell^{\prime}}$ within $\Phi_{\ell^{\prime}}$, and the take the disjunction of all such states. This is precisely captured by $\Phi_{\ell}=\vee\left\{\mu\left(\ell^{\prime}\right) \mid \ell^{\prime} \in\right.$ $\operatorname{pre}(\ell)\} ;(2)$ when $\ell$ is the first program point of a method $m \neq$ main, the most precise MHF state $\Phi_{\ell}$ that we can have, ignoring how $m$ was called, is that all local variables point to finished tasks (since they are mapped to $\perp$ when entering a method), and that the parameters might point to finished or active tasks which is precisely captured by $\Phi_{\ell}=\wedge\left\{x \mid x \in L_{m}\right\}$. But in addition we require that $m$ has actually been called - this is the role of add $\vee\left\{T F\left(\Phi_{\ell_{i}}\right) \mid \ell_{i} \in\left\{\ell_{1}, \ldots, \ell_{k}\right\}\right\}$; (3) the equation $\Phi_{\ell}=\wedge\left\{x \mid x \in L_{\text {main }}\right\}$, where $\ell$ is the entry of main, indicates that we start the execution from main.

Example 6. The following are the equations for the program points of $\mathrm{m}_{3}$ :

$$
\begin{array}{l|l|l}
\Phi_{26}=\mathrm{w} \wedge \mathrm{x} \wedge \mathrm{z} & \Phi_{29}=\oplus\left(\Phi_{28}, \mathrm{x}, \Phi_{59},\langle\rangle,\langle\rangle\right) & \Phi_{31}=\mathrm{w} \wedge \Phi_{30} \\
\Phi_{27}=\oplus\left(\Phi_{26}, \mathrm{z}, \Phi_{36},\langle\rangle,\langle\rangle\right) \vee \Phi_{31} & \Phi_{30}=\oplus\left(\Phi_{29}, \mathrm{w}, \Phi_{48},\langle\mathrm{x}, \mathrm{z}\rangle,\langle\mathrm{a}, \mathrm{b}\rangle\right) & \Phi_{32}=\Phi_{27} \\
\Phi_{28}=\Phi_{27} &
\end{array}
$$

Note, for example, the circular dependency between $\Phi_{27}$ and $\Phi_{31}$ which originates from the corresponding while loop. 
The next step is to solve $\mathcal{H}_{P}$, i.e., compute an MHF state $\Phi_{\ell}$, for each $\ell \in$ ppoints $(P)$, such that $\mathcal{H}_{P}$ is satisfiable. This can be done iteratively as follows. We start from an initial solution where $\Phi_{\ell}=$ false for each $\ell \in$ ppoints $(P)$. Then repeat the following until a fixed-point is reached: (1) substitute the current solution in the right hand side of the equations, and obtain new values for each $\Phi_{\ell}$; and (2) merge the new and old values of each $\Phi_{\ell}$ using $\vee$. E.g, solving the equation of Ex. 6, among other equations that were omitted, results in a solution that includes, among others, the MHF states of Ex. 4. In what follows we assume that $\mathcal{H}_{P}$ has been solved, and we use $\Phi_{\ell}$ to refer to the MHF state at $\ell$ in such solution.

Theorem 1. For any program point $\ell \in \operatorname{ppoints}(P)$, we have $\mathcal{F}(\ell) \subseteq \llbracket \Phi_{\ell} \rrbracket$.

In the rest of this article we let $\operatorname{mhf}_{\alpha}(\ell)=\left\{x \mid x \in V_{m}, \Phi_{\ell} \models x\right\}$, i.e., the set of finished future variable at $\ell$ that is induced by $\Phi_{\ell}$. Theorem 1 implies $\operatorname{mhf}_{\alpha}(\ell) \subseteq \operatorname{mhf}(\ell)$. Computing $\operatorname{mhf}_{\alpha}(\ell)$ using the MHF states of Ex. 4, among others that are omitted, results exactly in the MHF sets of Ex. 2.

\section{MHP Analysis}

In this section we present our MHP analysis, which is based on incorporating the MHF sets of Sec. 4 into the MHP analysis of [3]. In sections 5.1 and 5.2 we describe how we modify the two phases of the original analysis, and describe the gain of precision with respect to [3] in each phase.

\subsection{Local MHP}

The local MHP analysis (LMHP) considers each method $m$ separately, and for each $\ell \in \operatorname{ppoints}(m)$ it infers an LMHP state that describes the tasks that might be executing when reaching $\ell$ (considering only tasks invoked in $m$ ). An LMHP state $\Psi$ is a multiset of MHP atoms, where each atom represents a task and can be: (1) $y: \ell^{\prime}: \tilde{m}(\bar{x})$, which represents an active task that might be at any of its program points, including the exit one, and is bounded to future variable $y$. Moreover, this task is an instance of method $m$ that was called at program point $\ell^{\prime}$ (the calling site) with future parameters $\bar{x}$; or $(2) y: \ell^{\prime}: \hat{m}(\bar{x})$, which differs from the previous one in that the task can only be at the exit program point, i.e., it is a finished task. In both cases, future variables $y$ and $\bar{x}$ can be $\star$, which is a special symbol indicating that we have no information on the future variable.

Intuitively, the MHP atoms of $\Psi$ represent (local) tasks that are executing in parallel. However, since a variable $y$ cannot be bounded to more than one task at the same time, atoms bounded to the same variable represent mutually exclusive tasks, i.e., cannot be executing at the same time. The same holds for atoms that use mutually exclusive calling sites $\ell_{1}$ and $\ell_{2}$ (i.e., there is no path from $\ell_{1}$ and $\ell_{2}$ and vice versa). The use of multisets allows including the same atom several times to represent different instances of the same method. We let $(a, i) \in \Psi$ indicate that $a$ appears $i$ times in $\Psi$. Note that $i$ can be $\infty$, which happens when the atom corresponds to a calling site inside a loop, this guarantees convergence 
of the analysis. Note that the MHP atoms of [3] do not use the parameters $\bar{x}$ and the calling site $\ell^{\prime}$, since they do not benefit from such extra information.

Example 7. The following are LMHP states for some program points from Fig. 2:

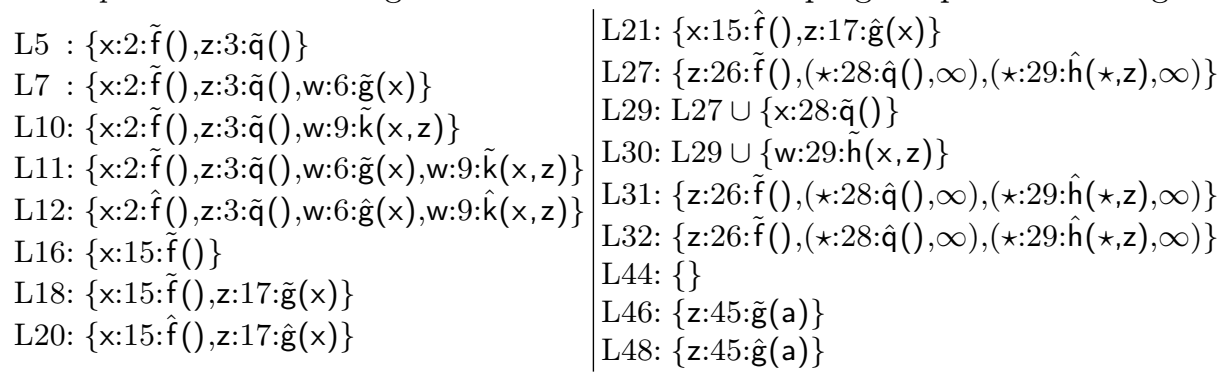

Let us explain some of the above LMHP states. The state at L5 includes $x: 2: \tilde{f}()$ and z:3: $\tilde{\mathrm{q}}()$ for the active tasks invoked at L2 and L3. The state at L11 includes an atom for each task invoked in $\mathrm{m} 1$. Note that those of $\mathrm{g}$ and $\mathrm{h}$ are bounded to the same future variable $w$, which means that only one of them might be executing at L11, depending on which branch of the if statement is taken. The state at L12 includes z:3: $\tilde{q}()$ since q might be active at L12 if we take the then branch of the if statement, and the other atoms correspond to tasks that are finished. The state at L27 includes z:26: $\tilde{f}()$ for the active task invoked at L26, and $\star: 28: \hat{q}()$ and $\star: 29: \hat{h}(\star, z)$ with $\infty$ multiplicity for the tasks created inside the loop. Note that the first parameter of $h$ is $\star$ since $x$ is rewritten at each iteration.

The LMHP states are inferred by a data-flow analysis which is defined as a solution of a set of LMHP constraints obtained by applying the following transfer function $\tau$ to the instructions. Given an LMHP state $\Psi_{\ell}$, the effect of executing instruction $I_{\ell}$ within $\Psi_{\ell}$, denoted by $\tau\left(I_{\ell}\right)$, is defined as follows:

- if $I_{\ell}$ is a call $y=m(\bar{x})$, then $\tau\left(I_{\ell}\right)=\Psi_{\ell}[y / \star] \cup\left\{y: \ell^{\prime}: \tilde{m}(\bar{x})\right\}$, which replaces each occurrence of $y$ by $\star$, since it is rewritten, and then adds a new atom $y: \ell: \tilde{m}(\bar{x})$ for the newly created task. E.g., the LMHP state of L30 in Ex. 7 is obtained from the one of L29 by adding w:29: $\tilde{h}(x, z)$ for the call at L29;

- if $I_{\ell}$ is await $y$ ?, and $\ell^{\prime}$ is the program point after $\ell$, then we mark all tasks that are bounded to a finished future variable as finished, i.e., $\tau\left(I_{\ell}\right)$ is obtained by turning each $z: \ell^{\prime \prime}: \tilde{m}(\bar{x}) \in \Psi_{\ell}$ to $z: \ell^{\prime \prime}: \hat{m}(\bar{x})$ for each $z \in \operatorname{mhf}_{\alpha}\left(\ell^{\prime}\right)$. E.g., the LMHP state of L12 in Ex. 7 is obtained from the one of L11 by turning the status of $\mathrm{g}, \mathrm{k}$, and $\mathrm{f}$ to finished (since $\mathrm{w}$ and $\mathrm{x}$ are finished at L12);

- otherwise, $\tau\left(I_{\ell}\right)=\Psi_{\ell}$.

The main difference w.r.t. the analysis of [3] is the treatment of await $y$ ?: while we use an MHF set computed using the inter-procedural MHF analysis of Sec. 4, In [3] the MHF set $\{y\}$ is used, which is obtained syntactically from the instruction. Our LMHP analysis, as [3], is defined as a solution of a set of LMHP constraints. In what follows we assume that the results of the LMHP analysis are available, and we will refer to the LMHP state of program point $\ell$ as $\Psi_{\ell}$. 


\subsection{Global MHP}

The results of the LMHP analysis are used to construct an MHP graph, from which we can compute the desired set of MHP pairs. The construction is exactly as in [3] except that we carry the new information in the MHP atoms. However, the process of extracting the MHP pairs from such graphs will be modified.

In what follows, we use $y: \ell: \breve{m}(\bar{x})$ to refer to an MHP atom without specifying if it corresponds to an active or finished task, i.e., the symbol $\breve{m}$ can be matched to $\tilde{m}$ or $\hat{m}$. As in [3], the nodes of the MHP graph consist of two method nodes $\tilde{m}$ and $\hat{m}$ for each method $m$, and a program point node $\ell$ for each $\ell \in \operatorname{ppoints}(P)$. Edges from $\tilde{m}$ to each $\ell \in$ ppoints $(m)$ indicate that when $m$ is active, it can be executing at any program point, including the exit, but only one. An edge from $\hat{m}$ to $\ell_{m}$ indicates that when $m$ is finished it can be only at its exit program point. The out-going edges from a program point node $\ell$ reflect the atoms of the LMHP state $\Psi_{\ell}$ as follows: if $\left(y: \ell^{\prime}: \breve{m}(\bar{x}), i\right) \in \Psi_{\ell}$, then there is an edge from node $\ell$ to node $\breve{m}$ and it is labeled with $i: y: \ell^{\prime}: \bar{x}$. These edges simply indicate which tasks might be executing in parallel when reaching $\ell$, exactly as $\Psi_{\ell}$ does.

Example 8. The MHP graphs $\mathcal{G}_{1}, \mathcal{G}_{2}$, and $\mathcal{G}_{3}$ in Fig. 2, correspond to methods $\mathrm{m}_{1}$, $\mathrm{m}_{2}$, and $\mathrm{m}_{3}$, each analyzed together with its reachable methods. For simplicity, the graphs include only some program points of interest. Note that the out-going edges of program point nodes coincide with the LMHP states of Ex. 7.

The procedure of [3] for extracting the MHP pairs from the MHP graph of a program $P$, denoted $\mathcal{G}_{P}$, is based on the following principle: $\left(\ell_{1}, \ell_{2}\right)$ is an MHP pair induced by $\mathcal{G}_{P}$ iff (i) $\ell_{1} \leadsto \ell_{2} \in \mathcal{G}_{P}$ or $\ell_{2} \leadsto \ell_{1} \in \mathcal{G}_{P}$; or (ii) there is a program point node $\ell_{3}$ and paths $\ell_{3} \leadsto \ell_{1} \in \mathcal{G}_{P}$ and $\ell_{3} \leadsto \ell_{2} \in \mathcal{G}_{P}$, such that the first edges of these paths are different and they do not correspond to mutually exclusive MHP atoms, i.e., they use different future variable and do not correspond to mutually exclusive calling sites (see Sec. 5.1). Edges with multiplicity $i>1$ represent $i$ different edges. The first (resp. second) case is called direct (resp. indirect) MHP, see Sec. 3.

Example 9. Let us explain some of the MHP pairs induced by $\mathcal{G}_{1}$ of Fig. 2. Since $11 \leadsto 35 \in \mathcal{G}_{1}$ and $11 \sim 58 \in \mathcal{G}_{1}$ we conclude that $(11,58)$ and $(11,35)$ are direct MHP pairs. Moreover, since these paths originate in the same node 11, and the first edges use different future variables, we conclude that $(58,35)$ is an indirect MHP pair. Similarly, since $11 \sim 38 \in \mathcal{G}_{1}$ and $11 \sim 50 \in \mathcal{G}_{1}$ we conclude that $(11,38)$ and $(11,50)$ are direct MHP pairs. However, in this case $(38,50)$ is not an indirect MHP pair because the first edges of these paths use the same future variable $w$. Indeed, the calls to $g$ and $k$ appear in different branches of an if statement. To see the improvement w.r.t. to [3] note that node 12 does not have an edge to $\tilde{f}$, since our MHF analysis infers that $x$ is finished at that L12. The analysis of [3] would have an edge to $\tilde{f}$ instead of $\hat{f}$, and thus it produces spurious pairs such as $(12,35)$. Similar improvements occur also in $\mathcal{G}_{2}$ and $\mathcal{G}_{3}$.

Now consider nodes 35 and 40 , and note that we have $11 \leadsto 35 \in \mathcal{G}_{1}$ and $11 \sim 40 \in \mathcal{G}_{1}$, and moreover these paths use different future variables. Thus, we 
conclude that $(35,40)$ is an indirect MHP pair. However, carefully looking at the program we can see that this is a spurious pair, because $\times$ (to which task $f$ is bounded) is passed to method $\mathrm{g}$, as parameter $\mathrm{w}$, and $\mathrm{w}$ is guaranteed to finish when executing await w? at L39. A similar behavior occurs also in $\mathcal{G}_{2}$ and $\mathcal{G}_{3}$. For example, the paths $30 \leadsto 58 \in \mathcal{G}_{3}$ and $30 \sim 40 \in \mathcal{G}_{3}$ induce the indirect MHP pair $(58,40)$, which is spurious since $\mathrm{x}$ is passed to $\mathrm{h}$ at L29, as parameter $\mathrm{a}$, which in turn is passed to $\mathrm{g}$ at L45, as parameter $\mathrm{w}$, and $\mathrm{w}$ is guaranteed to finish when executing await w? at L39.

The spurious pairs in the above example show that even if we used our improved LMHP analysis when constructing the MHP graph, using the procedure of [3] to extract MHP pairs might produce spurious pairs. Next we handle this imprecision, by modifying the process of extracting the MHP pairs to have an extra condition to eliminate such spurious MHP pairs. This condition is based on identifying, for a given path $\breve{m} \leadsto \ell \in \mathcal{G}_{P}$, which of the parameters of $m$ are guaranteed to finish before reaching $\ell$, and thus, any task that is passed to $m$ in those parameters cannot execute in parallel with $\ell$.

Definition 3. Let $p$ be a path $\breve{m} \leadsto \ell \in \mathcal{G}_{P}$, $\bar{z}$ be the formal parameter of $m$, and $I$ a set of parameter indices of method $m$. We say that $I$ is not alive along $p$ if (i) $p$ has a single edge, and for some $i \in I$ the parameter $z_{i}$ is in $\operatorname{mhf}_{\alpha}(\ell)$; or (ii) $p$ is of the form $\breve{m} \longrightarrow \ell_{1} \stackrel{i: k: y: \bar{x}}{\longrightarrow} \breve{m}_{1} \leadsto \ell$, and for some $i \in I$ the parameter $z_{i}$ is in $\operatorname{mhf}_{\alpha}\left(\ell_{1}\right)$ or $I^{\prime}=\left\{j \mid i \in I, z_{i}=x_{j}\right\}$ is not alive along $\breve{m}_{1} \leadsto \ell$.

Intuitively, $I$ is not alive along $p$ if some parameter $z_{i}$, with $i \in I$, is finished at some point in $p$. Thus, any task bounded to $z_{i}$ cannot execute in parallel with $\ell$.

Example 10. Consider $p \equiv \tilde{g} \leadsto 40 \in \mathcal{G}_{1}$, and let $I=\{1\}$, then $I$ is not alive along $p$ since it is a path that consists of a single edge and $w \in \operatorname{mhf}_{\alpha}(40)$. Now consider $\tilde{h} \leadsto 40 \in \mathcal{G}_{3}$, and let $I=\{1\}$, then $I$ is not alive along $p$ since $I^{\prime}=\{1\}$ is not alive along $\tilde{g} \leadsto 40$.

The notion of "not alive along a path" can be used to eliminate spurious MHP pairs as follows. Consider two paths

$$
p_{1} \equiv \ell_{3} \stackrel{i_{1}: y_{1}: \ell_{1}^{\prime}: \bar{w}}{\longrightarrow} \tilde{m}_{1} \leadsto \ell_{1} \in \mathcal{G}_{P} \text { and } p_{2} \equiv \ell_{3} \stackrel{i_{2}: y_{2}: \ell_{2}^{\prime}: \bar{x}}{\longrightarrow} \breve{m}_{2} \leadsto \ell_{2} \in \mathcal{G}_{P}
$$

such that $y_{1} \neq \star$, and the first node after $\tilde{m}_{1}$ does not correspond to the exit program point of $m_{1}$, i.e., $m_{1}$ is not finished and bounded to $y_{1}$. Define

- $F=\left\{y_{1}\right\} \cup\left\{y \mid \Phi_{\ell_{3}} \models y \rightarrow y_{1}\right\}$, i.e., the set of future variables at $\ell_{3}$ such that when any of them is finished, $y_{1}$ is finished as well; and

$-I=\left\{i \mid y \in F, x_{i}=y\right\}$, i.e., the indices of the parameters of $m_{2}$ to which we pass variables from $F$ (in $p_{2}$ ).

We claim that if $I$ is not alive along $p_{2}$, then the MHP pair $\left(\ell_{1}, \ell_{2}\right)$ is spurious. This is because before reaching $\ell_{2}$, some task from $F$ is guaranteed to terminate, and hence the one bounded to $y_{1}$, which contradicts the assumption that $m_{1}$ is not finished. In such case $p_{1}$ and $p_{2}$ are called mutually exclusive paths. 
Example 11. We reconsider the spurious indirect MHP pairs of Ex. 9. Consider first $(35,40)$, which originates from

$$
p_{1} \equiv 11 \stackrel{1: \mathrm{x}: 2:[]}{\longrightarrow} \tilde{f} \leadsto 35 \in \mathcal{G}_{1} \text { and } p_{2} \equiv 11 \stackrel{1: \mathrm{w}: 6:[\mathrm{x}]}{\longrightarrow} \tilde{g} \leadsto 40 .
$$

We have $F=\{\mathrm{x}, \mathrm{w}\}, I=\{1\}$, and we have seen in Ex. 10 that $I$ is not alive along $\tilde{g} \sim 40 \in \mathcal{G}_{1}$, thus $p_{1}$ and $p_{2}$ are mutually exclusive and we eliminate this pair. Similarly, consider $(58,40)$ which originates from

$$
p_{1} \equiv 30 \stackrel{1: \mathrm{x}: 28:[]}{\longrightarrow} \tilde{q} \leadsto 58 \in \mathcal{G}_{3} \text { and } p_{2} \equiv 30 \stackrel{1: \mathrm{w}: 29:[\mathrm{x}, \mathrm{z}]}{\longrightarrow} \tilde{h} \leadsto 40 .
$$

Again $F=\{\mathrm{x}, \mathrm{w}\}, I=\{1\}$, and we have seen in Ex. 10 that $I$ is not alive along $\tilde{h} \leadsto 40 \in \mathcal{G}_{3}$, thus $p_{1}$ and $p_{2}$ are mutually exclusive and we eliminate this pair.

Let $\tilde{\mathcal{E}}_{P}$ be the set of all MHP pairs obtained by applying the process of [3], modified to eliminate indirect pairs that correspond to mutually exclusive paths.

Theorem 2. $\mathcal{E}_{P} \subseteq \tilde{\mathcal{E}}_{P}$.

\section{Conclusions, Implementation and Related Work}

The main contribution of this work has been the enhancement of an MHP analysis that could only handle a restricted form of intra-procedural synchronization to the more general inter-procedural setting, as available in today's concurrent languages. Our analysis has a wide application scope on the inference of the main properties of concurrent programs, namely the new MHP relations are essential to infer (among others) the properties of the termination, resource usage and deadlock freedrom of programs that use inter-procedural synchronization.

Our analysis has been implemented in SACO [2], a Static Analyzer for $C$ oncurrent $O$ bjects, which is able to infer deadlock, termination and resource boundedness of ABS programs [10] that follow the concurrent objects paradigm. Concurrent objects are based on the notion of concurrently running objects, similar to the actor-based and active-objects approaches $[12,13]$. These models take advantage of the concurrency implicit in the notion of object to provide programmers with high-level concurrency constructs that help in producing concurrent applications more modularly and in a less error-prone way. Concurrent objects communicate via asynchronous method calls and use await instructions to synchronize with the termination of the asynchronous tasks. Therefore, the abstract model used in Sec. 2 fully captures the MHP relations arising in ABS programs.

The implementation has been built on top of the original MHP analysis in SACO. The MHF analysis has been implemented and its output has been used within the local and global phases of the MHP analysis, which have been adapted to this new input as described in the technical sections. The remaining analyses in SACO did not require any modification and now they work for interprocedural synchronization as well. Our method can be tried online at: http: //costa.ls.fi.upm.es/saco/web by enabling the option Inter-Procedural Synchronization of the MHP analysis in the Settings section. One can then 
apply the MHP analysis by selecting it from the menu for the types of analyses and then clicking on Apply. All examples used in the paper are available in the folder Forte15 adapted to the syntax of the ABS language. In the near future, we plan to apply our analysis to industrial case studies that are being developed in ABS but that are not ready for experimentation yet.

There is an increasing interest in asynchronous programming and in concurrent objects, and in the development of program analyses that reason on safety and liveness properties [6]. Existing MHP analyses for asynchronous programs $[3,11,1]$ lose all information when future variables are used as parameters, as they do not handle inter-procedural synchronization. As a consequence, existing analysis for more advanced properties [9,4] that rely on the MHP relations do all lose the associated analysis information on such futures. In future work we plan to study the complexity of our analysis, which we conjuncture to be in the same complexity order as [4]. In addition, we plan to study the computational complexity of deciding MHP, for our abstract models, with and without inter-procedural synchronizations in a similar way to what has been done in [5] for the problem of state reachability.

\section{References}

1. S. Agarwal, R. Barik, V. Sarkar, and R. K. Shyamasundar. May-happen-in-parallel analysis of x10 programs. In K. A. Yelick and J. M. Mellor-Crummey, editors, Proc. of PPOPP'0\%, pages 183-193. ACM, 2007.

2. E. Albert, P. Arenas, A. Flores-Montoya, S. Genaim, M. Gómez-Zamalloa, E. Martin-Martin, G. Puebla, and G. Román-Díez. SACO: Static Analyzer for Concurrent Objects. In Proc. of TACAS'14, volume 8413 of LNCS, pages 562567. Springer, 2014.

3. E. Albert, A. Flores-Montoya, and S. Genaim. Analysis of May-Happen-in-Parallel in Concurrent Objects. In Proc. of FORTE'12, volume 7273 of LNCS, pages 35-51. Springer, 2012.

4. E. Albert, A. Flores-Montoya, S. Genaim, and E. Martin-Martin. Termination and Cost Analysis of Loops with Concurrent Interleavings. In ATVA 2013, LNCS 8172, pages 349-364. Springer, October 2013.

5. A. Bouajjani and M. Emmi. Analysis of Recursively Parallel Programs. ACM Trans. Program. Lang. Syst., 35(3):10, 2013.

6. A. Bouajjani, M. Emmi, C. Enea, and J. Hamza. Tractable Refinement Checking for Concurrent Objects. In Proc. of POPL 2015, pages 651-662. ACM, 2015.

7. F. S. de Boer, D. Clarke, and E. B. Johnsen. A Complete Guide to the Future. In ESOP'07, LNCS 4421, pages 316-330. Springer, 2007.

8. C. Flanagan and M. Felleisen. The semantics of future and its use in program optimization. In 22nd ACM SIGPLAN-SIGACT Symposium on Principles of Programming Languages, 1995.

9. A. Flores-Montoya, E. Albert, and S. Genaim. May-Happen-in-Parallel based Deadlock Analysis for Concurrent Objects. In FORTE'13, LNCS, pages 273-288. Springer, 2013.

10. E. B. Johnsen, R. Hähnle, J. Schäfer, R. Schlatte, and M. Steffen. ABS: A Core Language for Abstract Behavioral Specification. In FMCO'10 (Revised Papers), LNCS 6957, pp. 142-164. Springer, 2012. 
11. J. K. Lee, J. Palsberg, R. Majumdar, and H. Hong. Efficient may happen in parallel analysis for async-finish parallelism. In In $S A S$ 2012, volume 7460, pages 5-23. Springer, 2012.

12. J. Schäfer and A. Poetzsch-Heffter. JCobox: Generalizing Active Objects to Concurrent Components. In Proc. of ECOOP'10, LNCS, pages 275-299. Springer, 2010.

13. S. Srinivasan and A. Mycroft. Kilim: Isolation-Typed Actors for Java. In Proc. of ECOOP'08, LNCS 5142, pages 104-128. Springer, 2008. 